\title{
The mechanisms of estrogen regulation of bone resorption
}

\author{
B. Lawrence Riggs
}

Endocrine Research Unit, Mayo Clinic and Foundation, Rochester, Minnesota 55905, USA. Phone: (507) 255-6788; Fax: (507) 284-8271; E-mail: riggs.lawrence@mayo.edu.

Estrogen (E) deficiency causes both the early and late forms of osteoporosis in postmenopausal women and contributes to the development of osteoporosis in elderly men (1). It is associated with large increases in bone resorption caused by increased osteoclast (OC) numbers (due to enhanced OC formation and reduced OC apoptosis) and by increased OC activity (2). Since the demonstration in 1988 that bone cells contain functional E receptors, progress on elucidating the molecular basis of $\mathrm{E}$ action has been rapid, albeit controversial and incomplete.

Early studies on E's role in bone metabolism focused on the role of the proinflammatory cytokines - IL-1, IL6 , TNF- $\alpha$, granulocyte macrophage colony-stimulating factor, macrophage colony-stimulating factor (M-CSF), and prostaglandin- $\mathrm{E}_{2}\left(\mathrm{PGE}_{2}\right)$. These factors increase bone resorption, mainly by increasing the pool size of pre-OCs in bone marrow $(2,3)$, and are downregulated by E. Moreover, ovariectomyinduced increases in OCs are attenuated or prevented by measures that impair the synthesis of or response to IL-1, IL-6, TNF- $\alpha$, or $\operatorname{PGE}_{2}(2,3)$. Other studies have found that $\mathrm{E}$ upregulates TGF- $\beta$, an inhibitor of bone resorption that acts directly on OC to decrease activity and increase apoptosis (2).

However, E regulation of bone resorption must now be re-evaluated in the light of the recent discovery of three new members of the TNF ligand and receptor signaling family that serve as the final effectors of OC differentiation and function $(4,5)$. The long-sought osteoblast-derived paracrine effector of OC differentiation has been identified as the receptor activator of NF- $\mathrm{KB}$ ligand (RANKL, also called OPG ligand or $\mathrm{OC}$ differentiating factor), which is expressed by stromal-osteoblast lineage cells. Contact between these cells and cells of the OC lineage allows RANKL to bind its physiologic receptor, RANK, potently stimulating all aspects of $\mathrm{OC}$ function: In response to RANKL signaling, OC differentiation and activity increase, and OC apoptosis decreases. Indeed, RANKL is both necessary and sufficient for OC formation, provided that permissive concentrations of $\mathrm{M}$ CSF are present. The stromalosteoblast lineage cells also secrete osteoprotegerin (OPG), a soluble decoy receptor that neutralizes RANKL. E increases OPG (5) and decreases M-CSF (3) and RANK (6). Part of the effect on this signaling system may be indirect, acting through E-responsive intermediaries. Thus, IL- 1 and TNF- $\alpha$ increase RANKL, OPG, and M-CSF, whereas $\mathrm{PGE}_{2}$ increases RANKL and decreases OPG $(3,5)$. E has not yet been shown to regulate RANKL directly.

In elegant studies published in this issue of the JCI, Cenci et al. (7) report

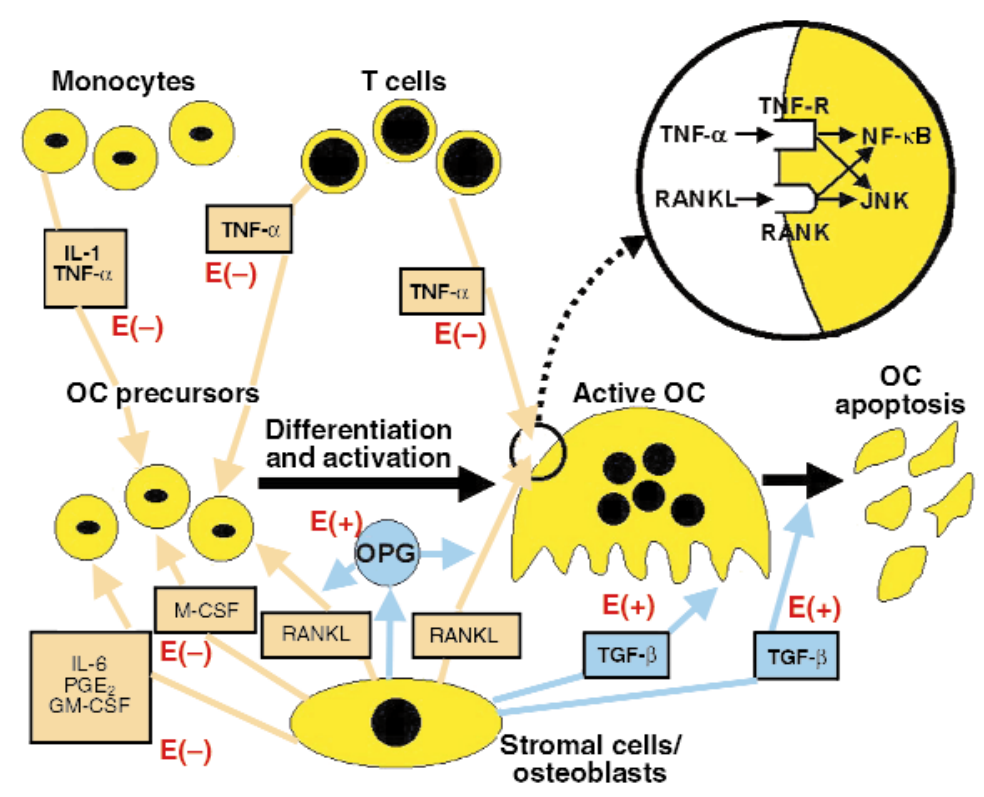

Figure 1

Major cytokines in the bone microenvironment that regulate OC function. Stimulatory factors are shown in orange and inhibitory factors are shown in blue. Positive (+) or negative (-) effects of $E$ on these regulatory factors are shown in red. The blow-up circle shows that TNF- $\alpha$ and RANKL act through separate receptors, but both activate the NF-KB and JNK intracellular signaling pathways. GM-CSF, granulocyte macrophage-colony-stimulating factor. 
stimulation of RANKL production (3, $5)$, since TNF- $\alpha$ fails to induce OC formation in BMMs from OVX mice lacking the 555 TNF- $\alpha$ receptor (TNF-R1). Cenci et al. (7) note that both RANKL and TNF- $\alpha$ independently activate the $\mathrm{NF}-\kappa \mathrm{B}$ and JNK intracellular signaling pathways in OC lineage cells, and they hypothesize that this convergence explains the additive effects of the two cytokines. They conclude that while MCSF and RANKL are essential for physiologic OC renewal, TNF- $\alpha$ plays a key causal role in the bone loss associated with E deficiency.

Although these data are important, several caveats must be kept in mind. First, the regulation of bone metabolism varies widely among rodents of different ages, strains, and species, and varies even more between rodents and humans, raising serious questions about the generality of the findings. Indeed, Pacifici's laboratory has previously reported that TNF- $\alpha$ and IL-1 must be inhibited simultaneously if bone loss is to be prevented in OVX rats (8). Also, Miyaura et al. (9) found that the combined effect of IL- $1 \alpha$, IL- 6 , and $\mathrm{PGE}_{2}$ could account for the increase in resorption bioactivity from marrow of another strain of OVX mice. Also, prevention of postovariectomy bone loss by blocking the production or activity of a single cytokine does not establish per se that it is the sole causal agent.
Because bone-regulating cytokines, such as IL-1, TNF- $\alpha$, and IL- 6 , synergize to stimulate their own and each other's synthesis, a small change in one cytokine in the bone microenvironment could dramatically alter the concentration of the others. Thus, the absence of any one cytokine may be sufficient to prevent this amplification from occurring. Finally, it seems unlikely that TNF- $\alpha$ is the sole mediator of the $\mathrm{E}$ effect on bone resorption, because genetically targeted mice deficient in TNF-R1 have normal bone histology (10). In contrast, M-CSF, OPG, and RANKL are potent final effectors that can induce the extremes of skeletal changes - osteoporosis or osteopetrosis - when their gene is overexpressed or deleted $(4,5)$. Thus, these factors should be able to compensate reciprocally for the effect of changes in upstream cytokines. That this does not occur suggests that E deficiency affects them as well.

It seems more likely that $\mathrm{E}$ inhibits bone resorption by inducing small but cumulative changes in multiple Edependent regulatory factors, as shown in Figure 1. Of the E-dependent factors affecting OC formation, TNF- $\alpha$ and the OPG/RANKL/RANK system may be most important, whereas TGF- $\beta$ and the OPG/RANKL/RANK system may have greater effects on OC activity and apoptosis. Clearly, more data are need- ed, especially on cytokine changes in the bone microenvironment of women with early postmenopausal bone loss or postmenopausal osteoporosis.

1. Riggs, B.L., Khosla, S., and Melton, L.J., III. 1998. A unitary model for involutional osteoporosis: estrogen deficiency causes both type I and type II osteoporosis in postmenopausal women and contributes to bone loss in aging men. J. Bone Miner. Res. 13:763-773.

2. Manolagas, S.C. 2000. Birth and death of bone cells: basic regulatory mechanisms and implications for the pathogenesis and treatment of osteoporosis. Endocr. Rev. 21:115-137.

3. Pacifici, R. 1996. Estrogen, cytokines, and pathogenesis of postmenopausal osteoporosis. J. Bone Miner. Res. 11:1043-1051.

4. Suda, T., et al. 1999. Modulation of osteoclast differentiation and function by the new members of the tumor necrosis factor receptor and ligand families. Endocr. Rev. 20:345-357.

5. Hofbauer, L.C., et al. 2000. The roles of osteoprotegerin and osteoprotegerin ligand in the paracrine regulation of bone resorption. J. Bone Miner. Res. 15:2-12.

6. Shevde, N.K., Bendixen, A.C., Dienger, K.M., and Pike, J.W. 2000. Estrogens suppress RANK ligandinduced osteoclast differentiation via a stromal cell independent mechanism involving c-Jun repression. Proc. Natl. Acad. Sci. USA. 97:7829-7834.

7. Cenci, S., et al. 2000. Estrogen deficiency induces bone loss by enhancing T cell production of TNF$\alpha$. J. Clin. Invest. 106:1229-1237.

8. Kimble, R.B., et al. 1995. Simultaneous block of interleukin-1 and tumor necrosis factor is required to completely prevent bone loss in the early postovariectomy period. Endocrinology. 136:3054-3061.

9. Miyaura, C., et al. 1995. Endogenous boneresorbing factors in estrogen deficiency: cooperative effects of IL-1 and IL-6. J. Bone Miner. Res. 10:1365-1373.

10. Vargas, S.J., et al. 1996. Interleukin-6 expression and histomorphometry of bones from mice deficient in receptors for interleukin-1 or tumor necrosis factor. J. Bone Miner. Res. 11:1736-1744. 